\title{
Noise reduction of transient electromagnetic measurement data
}

\author{
Xin $W u$ \\ Institute of Electronics, CAS \\ Beijing, China
}

\begin{abstract}
The bipolar synchronous sampling method is widely used for noise reduction in Transient Electromagnetic Measurement. However, in practice the ability of this method is not enough to reduce the strong noise from multi-sources. In this paper, we propose an improved method for optimization of the Instrument Sampling Function to improve the capability of the System against the narrowband noise, specially the power line noise. A Gauss function is designed and used as a window function for corresponding gate function to improve the Noise reducing ability in broadband zone and another Gauss function filtered by a notching filter is designed and used as a window function for weighting function in order to improve the Noise reducing ability in narrowband zone. Finally we compared the results of data processing with the different instrument sampling functions before and after the Optimization, and it proved that this new method here has stronger ability in noise reduction for TEM Measurement.
\end{abstract}

Keywords-PRBS; system identification; electromagnetic impulse response

\section{INTRODUCTION}

TEM observation noise is broadly classified into the external and internal instrument noise. The internal noise of the instrument is mainly derived from magnetic field sensors. The noise's intensity level affects the accuracy and depth of TEM observation.

In practice, there may be some deviation between the actual frequency and the theoretical value, choosing emission period according to theoretical power frequency can't reduce the practical actual frequency and its harmonic well. In order to enhance the ability of noise reduction of the system, foreign scholars carried out research on the optimization of the bipolar synchronous sampling method. Macnae et al. proposed a method that weight and stack each period ${ }^{[1]}$.

In this paper, further optimization of the bipolar synchronous sampling method is proposed for better reduction effect for the actual power frequency. We compared the results of data processing with the different instrument sampling functions before and after the optimization, and it proved that this new method here has stronger ability in noise reduction for TEM Measurement.

\author{
Guoqiang Xue \\ Institute of Geology and Geophysics, CAS \\ Beijing, China
}

\section{The Noise Reduction TheOry OF THE BIPOLAR SYNCHRONOUS SAMPLING METHOD}

In traditional TEM synchronous sampling method, the process of output result can be expressed like that:

$$
y(t)=\int_{0}^{t} x(\theta) S(\theta) d \theta
$$

In this formula, $\mathrm{x}$ denotes TEM observation data; $\mathrm{y}$ denotes the final output value of a single gate that has been multiperiod stacked. The above function $\mathrm{S}$ denotes the Instrument Sampling Function(ISF) of a single gate, its definition can be expressed in this formula ${ }^{[1]}$

$$
S(t)=R(t) W(t)
$$

In this formula, $\mathrm{R}(\mathrm{t})$ is the reference function, $\mathrm{W}(\mathrm{t})$ is the weighting function.

The variance $\sigma^{2}$ of the output value $y(t)$ of gate in formula(1) can be expressed in below formula:

$$
\sigma^{2}=\int_{0}^{\infty} K^{2}(f)|n(f)|^{2} d f
$$

In which, K(f) denotes Noise Spectral Sensitivity Function; $\mathrm{n}(\mathrm{f})$ denotes the unilateral spectrum density of stable random noise. The relation between the Noise Spectral Sensitivity Function, $\mathrm{K}(\mathrm{f})$ and the Fourier transform of $\mathrm{s}(\mathrm{t}), \mathrm{S}(\mathrm{f})$ is that:

$$
K^{2}(f)=S(f) S^{*}(f)
$$

In this formula, $\mathrm{S}^{*}(\mathrm{f})$ is the conjugation of $\mathrm{S}(\mathrm{f})$.

\section{THE EXPLICIT IMPROVEMENT METHOD OF SAMPLING FUNCTION}

In order to optimize ISF, the first step is to research the building process and spectrum characteristics of ISF. Thus, a bipolar impulse pair whose period is T should be built:

$$
I_{I}(t)=\frac{1}{2}\left[\delta(t)-\delta\left(t-\frac{T}{2}\right)\right]
$$

Function Shah whose period is $\mathrm{T}$ :

$$
I I I_{T}(t)=\sum_{n=-\infty}^{\infty} \delta(t-n T)
$$


Convoluting II and IIIT, a bipolar impulse sequencer whose period is T can be built:

$$
I I I_{+/-}(t)=I_{I}(t) * I I I_{T}(t)=\frac{1}{2}\left[I I I_{T}(t)-I I I_{T}\left(t-\frac{T}{2}\right)\right]
$$

Its Fourier transform is that:

$$
F I I I_{+-}\left(f_{0}\right)=\frac{f_{0}}{2} I I I_{f_{0}}(f)\left(1-e^{-j \pi f T}\right)
$$

In this formula, $\mathrm{f} 0=1 / \mathrm{T}$. Normalizing (8) and converting it to time domain:

$$
I I I_{U+-}(t)=\frac{T}{2}\left[I I I_{T}(t)-I I I_{T}\left(t-\frac{T}{2}\right)\right]
$$

Equation (9) denotes a bipolar impulse sequence whose period is $\mathrm{T}$, its frequency spectrum is a unit amplitude impulse sequence. Then, designing a gate whose mid time is $c$, duration is $1 / \Delta$ in a single period (the traditional design of sampling tract):

$$
G(t)=\frac{1}{\Delta} \Pi\left(\frac{t-c}{\Delta}\right)
$$

In this formula, $\Pi$ denotes rectangular pulse. The Fourier transform of above gate function is that:

$$
F G(f)=\operatorname{sinc}(\Delta f) e^{-2 j \pi c f}
$$

The reference function can be obtained by convoluting (9) and (10):

$$
\begin{gathered}
R(t)=G(t) * I I I_{+/-}(t) \\
F R(f)=\frac{1}{2} I I I_{f_{0}}\left(1-e^{-j \pi f T}\right) \operatorname{sinc}(\Delta f) e^{-j \pi c f}
\end{gathered}
$$

FR(f) is the Fourier transform of $\mathrm{R}(\mathrm{t})$. Being similar with the definition of $G(t)$, assumed the number of stacking period is $\mathrm{N}$, the stack weight function $\mathrm{W}(\mathrm{t})$ can be defined:

$$
\begin{aligned}
W(t) & =\frac{1}{Q} \Pi\left(\frac{t-w}{Q}\right) \\
F W(f) & =\operatorname{sinc}(Q f) e^{-2 j \pi w f}
\end{aligned}
$$

In these formula, $\mathrm{Q}=\mathrm{NT}, \mathrm{w}$ is the mid time point of the total observation time length, FW(f) is the Fourier transform of w(t). Bringing (12) and (14) into (2).The ISF of traditional bipolar synchronous sampling method can be obtained and it can be denoted as SBB, in which S denotes ISF, the first letter of the subscript denotes the type of function that the sampling function uses, the second letter of the subscript denotes the type of function that the weighting function uses, SBB denotes the gate function and the weighting function all use rectangular (Box) pulse function.

The optimized sampling function and stack function can be built as below:

$$
\begin{gathered}
G_{G}(t)=\frac{1}{\varepsilon_{g} \sqrt{\pi}} e^{-\left(t / \varepsilon_{g}\right)^{2}} \\
W_{G}(t)=\frac{1}{Q} \Pi\left(\frac{t-w}{Q}\right) * \frac{1}{\varepsilon_{w} \sqrt{\pi}} e^{-\left(t / \varepsilon_{w}\right)^{2}}
\end{gathered}
$$

$\mathrm{GG}(\mathrm{t})$ is the optimized reference function and $\mathrm{WG}(\mathrm{t})$ is the optimized weighting function.

In order to researching the improvement effect of using $\mathrm{GG}(\mathrm{t})$ and $\mathrm{WG}(\mathrm{t})$ respectively, firstly, using (16) to instead (12) and bringing (14) into (2), then the ISF that only being optimized for sampling function can be obtained, the ISF can be donated as SGB, in which the subscript $G$ denotes the sampling function that has been improved with Gauss function, the subscript B denotes the weighting function that still uses rectangular (Box) pulse function.

After that, replacing (14) with (17) and bringing (16) and (17) into (2), then optimizing ISF with sampling function and weighting function, the optimized ISF is denoted as SGG which expressed the sampling function and the weighting function are all optimized with Gauss function.

SGG is a ISF optimized method that proposed by Nyboe.In the early research stage of this paper, SGG mothed is realized and tested in actual conditions. The result indicates that SGG method doesn't have good ability of noise reduction under some actual conditions. On the on hand, in practice, it is often to need to carry out work in the mining areas whose power frequency noise is strong when working. On this condition, SGG method doesn't have good ability of noise reduction for power frequency noise; On the other hand, when the deviation of the actual power frequency and the theory power frequency is large enough (more than $0.2 \mathrm{~Hz}$ ), SGG method's ability of noise reduction for power frequency will decline remarkably.

Therefore, in this paper, based on SGG, a new optimized method, SGN, is proposed. SGN uses all phase method and builds a notching filter (f) of $50 \mathrm{~Hz}$ and its odd harmonic. Using formula (17) for further optimization of weighting function $\mathrm{Wf}(\mathrm{t})$ :

$$
W_{f}(t)=W_{G}(t) * f
$$

Bringing (16) and (18) into (2), ISF whose ability of noise reduction has been strengthened can be obtained. The ISF can be donated as SGN, in which the subscript G denotes that the sampling function has been improved by Gauss function and the subscript $\mathrm{N}$ denotes that the weighting function has been improved by notching filter.

\section{THE COMPARISON OF IMPROVEMENT EFFECT}

Firstly, comparing SBB and SGB, Fig.1 is the pattern's comparison of SBB and SGB in time domain. As shown in Fig 1(a), the gate function of SBB is rectangular pulse function and that of SGB is Gauss function. Fig. 1(b) describes the stack weight of all the 26th gates in the whole observation time. It indicates that the stack weight of any period of SBB and SGB are the same. Comparing SBB and SGB in frequency domain, as shown in Fig. 2, SGB has a better ability of noise reduction because of the use of Gauss function. It indicates that although 
Gauss function has been used to optimize the gate function, these two kinds of ISF have the same ability of noise reduction for signal whose frequency is $50 \mathrm{~Hz}$. Thus, the optimization of gate function can enhance system's ability of noise reduction for high frequency noise.

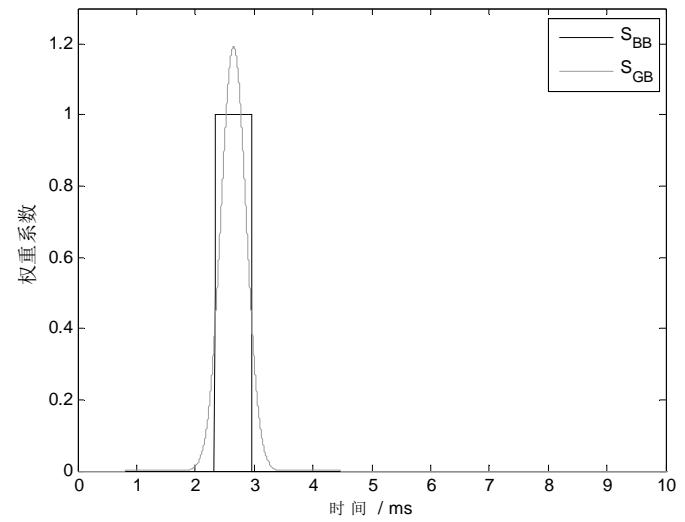

(a)

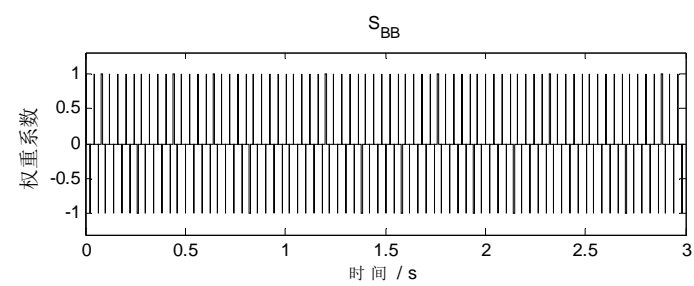

$\mathrm{s}_{\mathrm{GB}}$

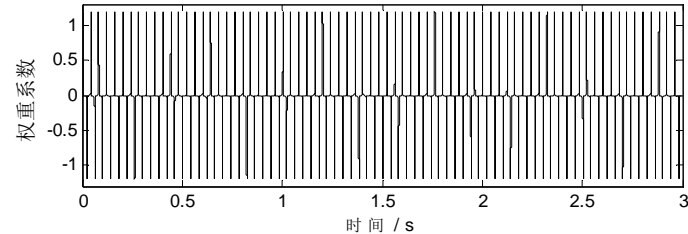

(b)

Fig. 1. time sequence comparison between SBB and SGB (a) Gate function comparison between SBB and SGB; (b)Weighting function comparison between SBB and SGB

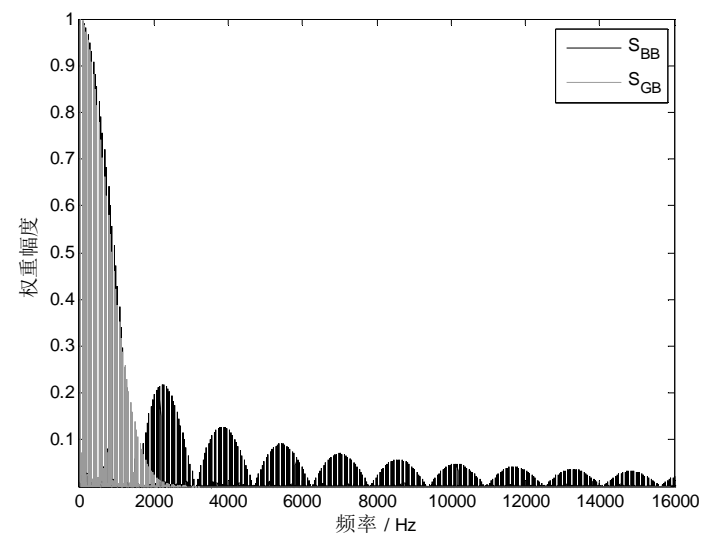

Fig. 2. comparison of single-sided amplitude spectrum between SBB and SGB
Fig. 3 is time sequence comparison between SGB and SGG. As shown in Fig 3(a), the gate function of SGB and SGG are all Gauss function. Fig. 3(b) indicates that in the whole observation time, the stack weight of all the periods of SGB are the same. However, those of SGG are different and its envelope curve expresses Gauss function's pattern.

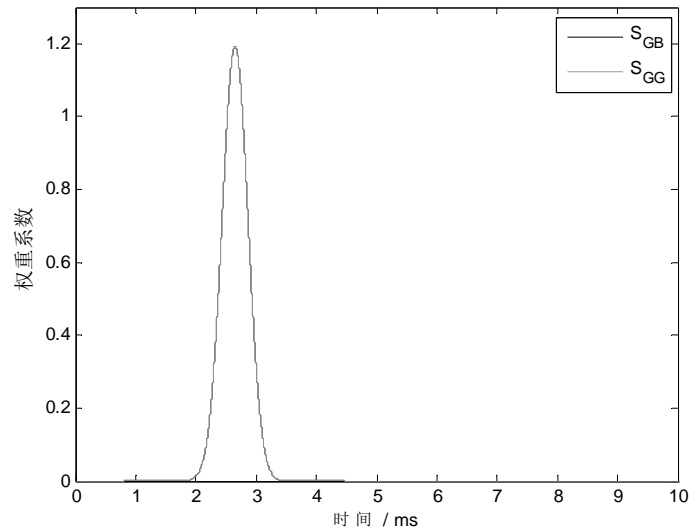

(a)
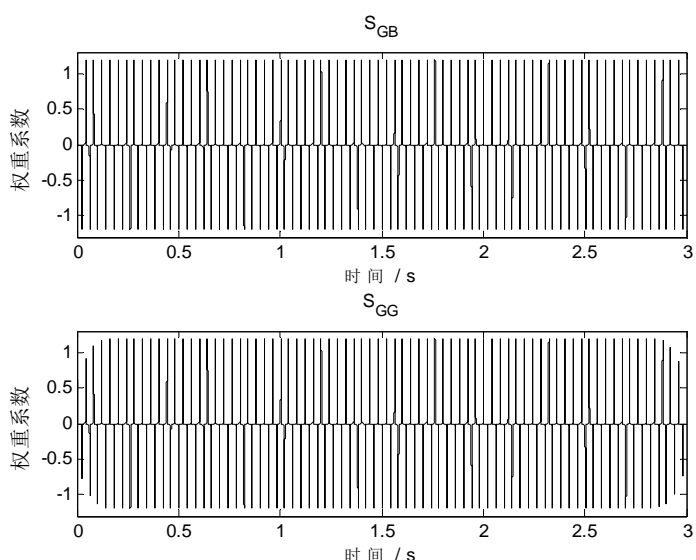

(b)

Fig. 3. time sequence comparison between SGB and SGG

(a) Gate function comparison between SGB and SGG;

(b) Weighting function comparison between SGB and SGG

Comparing SGB and SGG in frequency domain, as shown in Fig. 4(a), SGB and SGG have a better ability of noise reduction in the high frequency part because of the use of Gauss function. Fig 4.(b) is the low frequency part of Fig. 4(a) in the single-logarithmic coordinate. It indicates that because of the optimization for each period's stack weight, the SGG's ability of noise reduction for narrow band noise is better than that of SGB. Thus, optimizing the weighting function can enhance system's ability of noise reduction for low frequency narrow band noise. 


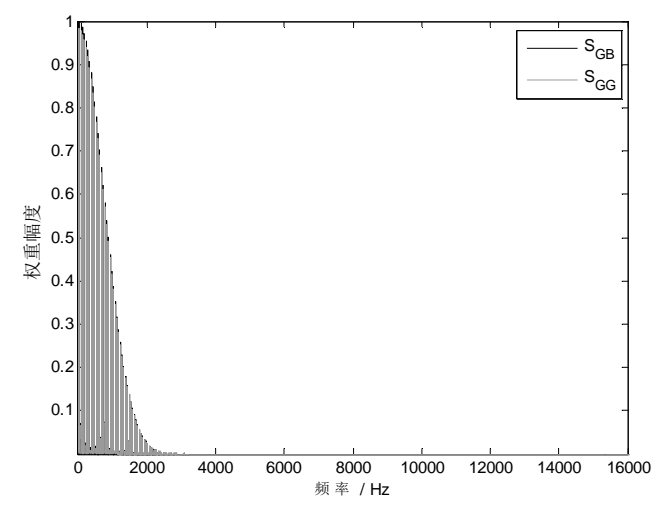

(a)

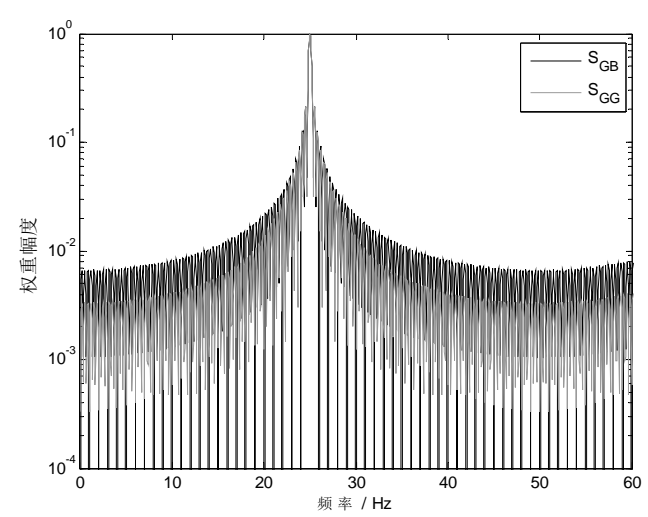

(b)

Fig. 4. comparison of single-sided amplitude spectrum between SGB and SGG

(a) Comparison of single-sided amplitude spectrum between SGB and SGG in broadband; (b) Comparison of single-sided amplitude spectrum between SGB and SGG in narrowband

At last, comparing SGG and SGN. As shown in Fig 5(a) Gauss function is also used on the gate of SGN, so SGN has the same ability of noise reduction for high frequency noise. Fig 5(b) is the low frequency part of Fig 5(a) in the singlelogarithmic coordinate. It indicates that there is a trap wave window whose width is about $1.2 \mathrm{~Hz}$ at the $50 \mathrm{~Hz}$ in SGN, for which it has a stranger ability of noise reduction for power frequency.

By using Gauss function on gate, this method endows suitable stack weight to each sampling points in gate to enhance system's ability of noise reduction for broadband

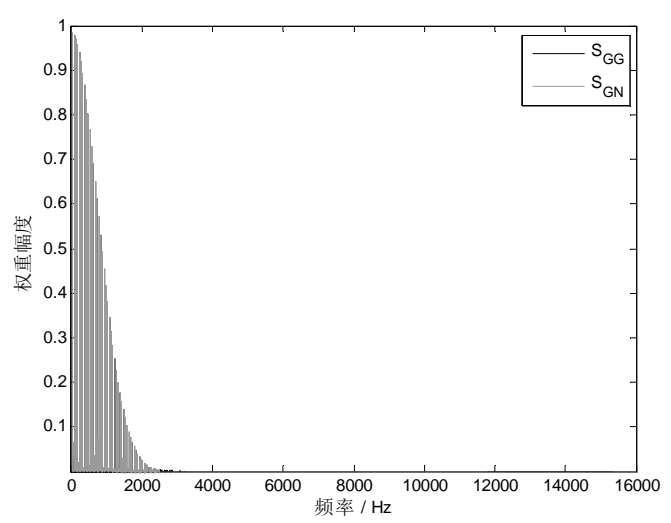

(a)

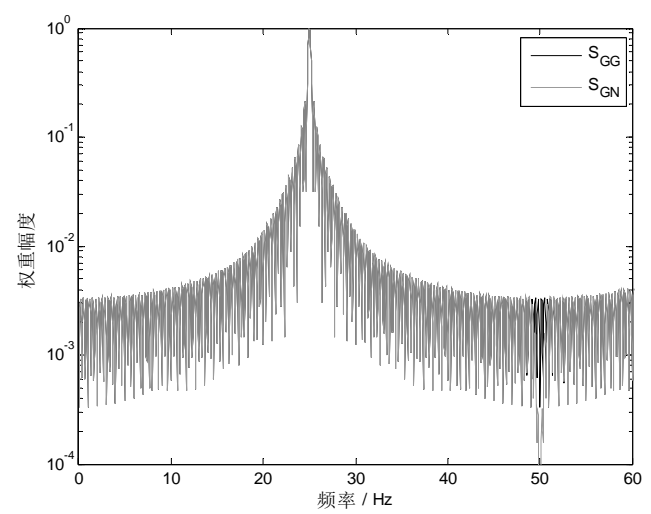

(b)

Fig. 5. comparison of single-sided amplitude spectrum between SGG and SGN

(a) Comparison of single-sided amplitude spectrum between SGG and SGN in broadband; (b) Comparison of single-sided amplitude spectrum between SGG and SGN in narrowband

noise. Complex window function is used on each stack period in observation sequence to improve the stack weight of each period, which can enhance system's ability of noise reduction for actual power frequency noise. Using traditional bipolar synchronous sampling method SBB, Comparing with traditional SBB method, the ability of noise reduction of SGN is enhanced $46 \%$. Thus, the method proposed by this paper has stronger ability of noise reduction than traditional method.

\section{REFERENCES}

[1] McCracken K G, Pik J P, Harris R W. 1984. Noise in EM Exploration Systems. Exploration Geophysics: 15, 169-174J. Clerk Maxwell, A Treatise on Electricity and Magnetism, 3rd ed., vol. 2. Oxford: Clarendon, 1892, pp.68-73. 